\title{
OPERATION AND RECENT DEVELOPMENTS AT THE ESRF
}

\author{
P Berkvens, J.C. Biasci, J. Chavanne, P Duru, P. Elleaume, L Farvacque, T Günzel, L. Hardy, \\ J Jacob, R Kersevan, G Naylor, Y Papaphilipou, E. Plouviez, J.L. Revol, A. Ropert, K Scheidt, \\ ESRF, Grenoble, France
}

\section{Abstract}

The ESRF has been operating for more than 10 years. As a result of the maintenance policy a high level of beam availability as well as a long beam time between failures has been maintained. In addition, a continuous effort is made to develop the machine further. The operation status and the experience gained with the recent upgrades are reported. It concerns:

- The successful operation with 5 in-vacuum undulators operated at a 5-6 mm magnetic gap.

- The implementation of a new hybrid type filling pattern which combines groups of bunches at full current and single bunch.

- The successful qualification of a 5 metre -long, $8 \mathrm{~mm}$ vertical aperture insertion device (ID) aluminum chamber coated with non evaporable getter material.

- The evolution of the impedance with associated consequences on instability thresholds, following the installation of a number of low aperture insertion device chambers.

- The successful test of the SOLEIL superconducting cavity.

- The injection front end open in order to ensure a better thermal stability in the beamlines.

\section{THE ESRF IN 2003}

The European Synchrotron Radiation Facility (ESRF) located in Grenoble, France is a joint facility supported and chaired by 17 Countries. This third generation storage ring X-ray light source has been in routine operation for more than 10 years. The source delivers 5500 hours of beam to 43 beamlines simultaneously.

\begin{tabular}{|l|c|c|}
\hline Particles & Electrons & \\
\hline Energy & 6.03 & $\mathrm{GeV}$ \\
\hline Circumference & 844 & $\mathrm{~m}$ \\
\hline Multibunch Current & 200 & $\mathrm{~mA}$ \\
\hline Lifetime (Uniform fill) & 80 & Hours \\
\hline Horizontal emittance & 4 & $\mathrm{~nm} . \mathrm{rad}$ \\
\hline Coupling & 0.6 & $\%$ \\
\hline 16 Bunch (Single) current & $90(16)$ & $\mathrm{mA}$ \\
\hline
\end{tabular}

Table 1: Main parameters

A large variety of insertion devices is installed in the 27 available straight sections:

- 64 in-air IDs (two or three 1.7 metre-long segments per straight section)

- 5 in-vacuum undulators (2 metre-long) [1]

The bending magnet radiation is now used by 15 beamlines.

\section{OPERATION IN 2002}

The figures of merit for 2002, namely the mean time between failure (MTBF) and availability, were maintained at the same level as in 2001. Out of the 5495 hours scheduled for beam delivery to the Users (USM), 5319 hours were delivered, which represents an availability of $96.8 \%$. The remaining hours are shared between the dead time for refills: 67 hours for 507 refills (i.e., $1.2 \%$ of USM time) and the time lost due to failures: 110 hours (i.e. $2 \%$ of USM time). With 95 beam interruptions, the MTBF reached a record of 57.8 hours, which confirms the improvement of this figure of merit obtained in 2001 (see Fig 1). Another way to appreciate these figures is to bear in mind that there were 17 periods with more than 72 hours of delivery without a single failure, out of which 9 periods were greater than 120 hours! The efforts put by all groups on preventive maintenance and failure analysis has largely contributed to the continuous increase of the MTBF and availability during the last few years.

It is worth pointing out that these results, and in particular the low rate of failures, were maintained whilst a great deal of developments were under way: in particular, the successful tests of the Soleil superconducting cavity installed throughout 2002. These tests were performed without any change of the beam size, bunch length and with the same stability. Indeed, this was fully transparent to user operation.

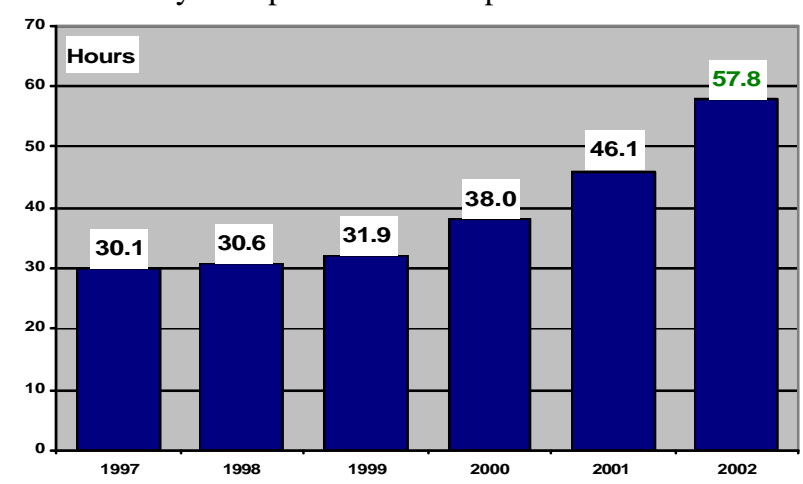

Fig. 1: Evolution of the Mean Time Between Failures.

Multibunch filling pattern, which represents $67 \%$ of the total user time, was mostly delivered in uniform filling with a lifetime greater than 70 hours at $200 \mathrm{~mA}$. Despite a slightly lower lifetime, the $2 * 1 / 3$ fill is still used for time structure experiments. The rest is shared between the 16 bunch, single bunch and hybrid modes. The deterioration of the horizontal beam dynamics was clearly observed during last year, making the preparation of $20 \mathrm{~mA}$ single bunch and hybrid modes more difficult. 


\section{INJECTION WITH FRONT END OPEN}

This year, the operation of the ESRF beamlines was dramatically changed by the implementation of the injection with front-ends open, allowing real continuous operation. Before, the shutters of all beamlines were closed for a few minutes during injection, resulting in large temperature variations on the optics which caused interruptions of data acquisition up to an hour after each injection. Leaving the shutters open during injection has dramatically improved the situation.

This mode of operation has to be compatible with the present ESRF radiation protection policy, which stipulates that all staff working at the ESRF are considered as non-exposed workers. This implies that the dose limits for the public, as defined in the Euratom/9629 decree, do not exceed $1 \mathrm{mSv}$ per year (or $2 \mu \mathrm{Sv}$ over 4 hours if taking into account working time). Radiation monitors interlock the beam in the linac if the dose exceeds the limit. The accidental steering of a single $1 \mu \mathrm{s}$ electron beam pulse injected from the booster into a beamline with open front end would result in an integrated dose, largely exceeding the $2 \mu \mathrm{Sv}$ limit. No interlocked radiation monitor could therefore be fast enough to protect against such an accidental situation. A detailed study has demonstrated the impossibility of steering beam from the booster into a beamline if there is already a stored beam. A dedicated and redundant beam current monitor has been developed and integrated in the machine personnel safety system. Injection with FE open is only allowed if this current monitor measures a stored beam larger than $5 \mathrm{~mA}$. During the first one and a half months of operation in this mode, uniform, 16 bunch, single bunch and $2 \times 1 / 3$ filling modes were delivered. The evolution of the dose over 4 hours measured around all beamline optics hutches indicated that the injection losses, as well as the losses due to bunch cleaning in all filling modes, produce very low dose rates compatible with the limit. The measured dose is limited by the background level in the Experimental Hall (which varies between 80 and $100 \mathrm{nSv} \cdot \mathrm{h}^{-1}$ ), with a small contribution from scattered bremsstrahlung, which depends on the type of filling mode.

On the machine side, the implementation of the Injection FE Open as a standard mode of operation was rather straightforward. The main modifications concerned the logical unit of machine protection system and the implementation of a software counter indicating the remaining time before the next injection. The value of the counter can be used to synchronise the data acquisition. In order for the beamlines to fully benefit from Front Ends Open injection, it is essential to maintain the insertion device gap unchanged during injection. Since in-vacuum undulators (built with $\mathrm{Sm}_{2} \mathrm{Co}_{17}$ magnets, more resistant than $\mathrm{NdFeB}$ ) are the most sensitive to radiation damage when exposed to high energy electrons, their minimum gap is presently limited to $8 \mathrm{~mm}$ (instead of $6 \mathrm{~mm}$ ) during injection [1]. For the corresponding beamlines, this generates a maximum $30 \%$ heatload variation for a few minutes. In the long term, it is planned to reduce this gap limit following a detailed quantitative study performed on ID6, the machine beamline.

User feedback was extremely positive despite the constraints imposed on the beamlines using in-vacuum devices. The strong reduction of thermal variation and the possibility to use the beam during injection result in an increase of beam availability for most beamlines. In multibunch modes with long lifetimes (50-90 hrs), the injection with front ends open twice a day is indeed close to ideal for the beamlines. However, it is less favourable in time structure modes where the reduced lifetime imposes more frequent injections. Moreover, the cleaning procedure renders the beam unavailable for a few minutes during each injection. Work has been initiated to improve this by performing the cleaning in the booster.

\section{TEST OF A NEW HYBRID MODE}

A new hybrid mode has been developed and was delivered for 8 hours during Machine Dedicated Time to Users who volunteered to test it. It consists of 24 groups of 8 bunches ( $1 \mathrm{~mA}$ each) with an additional single bunch of $4 \mathrm{~mA}$. The lifetime at $200 \mathrm{~mA}$ reached 26 hours; other beam parameters were close to the usual multibunch figures. Following the positive feedback received from Users, this mode will be proposed in replacement of the standard hybrid mode (9\% of Lser time in 2002). The difficulties encountered with this new mode concern the cleaning of the parasitic bunches. Firstly, due to the low current per bunch, the detuning of the vertical betatron frequency induced by the machine impedance is small. Secondly, there is a strong quadrupolar detuning as a function of the total beam current induced by the low gap chamber resistivity and its asymmetry [2]. The precise definition of the cleaning frequency is obtained by injecting a few $\mathrm{mA}$ of multibunch beam. The excitation of the low populated bunches is performed at a higher order coupled bunch mode, using a stripline shaker to reduce the excitation of the main bunches through the narrow band resistive wall impedance.

\section{INTENSITY RAMPING TO 250 mA}

A beam current of $250 \mathrm{~mA}$ was stored in the machine for the first time in 2001. However, the radiation induced outside the shielding exceeded the authorised level and tests were temporarily interrupted. After a campaign of systematic shielding reinforcement, tests resumed in April 2003. Stable beam at $250 \mathrm{~mA}$ was stored in $1 / 3$ and $2 / 3$ filling modes. No abnormal pressure rise or temperature increase on critical components was observed. The lifetime reached 44 hours in $2 / 3$ filling (to be compared to 60 hours at $200 \mathrm{~mA}$ ). However, in uniform filling, the current was limited to $240 \mathrm{~mA}$ due to a transverse High Order Mode instability that still needs to be mastered. This is the first observation of an instability due to a transverse HOM at the ESRF! Further studies are planned 
to characterize and fully master the beam parameters at $250 \mathrm{~mA}$.

\section{PROGRESS WITH NEG COATED CHAMBERS}

The installation of non-evaporable getter (NEG) coated vessels continued throughout the year. The main development was the production of a prototype followed by full tests of an $8 \mathrm{~mm}$ aperture 5 metre long aluminium vessel. The choice of aluminium instead of stainless steel was made to reduce both manufacturing costs and the excitation of the resistive wall instability. However, raw aluminium presents strong synchrotron radiation induced desorption compared to stainless steel. The conditioning of the prototype chamber (without NEG coating) installed in May 2002 was so slow and the bremsstrahlung produced was so high that the chamber was removed immediately after installation. When the same chamber coated with NEG was re-installed in October 2002 a rapid conditioning was observed (half the nominal multibunch lifetime was obtained after 20 minutes of beam at full current). The coating of the tiny 57 x $8 \mathrm{~mm}$ elliptical chamber was made at CERN. A NEG coating facility is being developed at the ESRF, which should allow the coating of 5 metre-long insertion device vessels.

\section{EVOLUTION OF MACHINE IMPEDANCE AND INSTABILITY THRESHOLDS}

The vertical and horizontal transverse impedance of most of the machine components have been determined using the electromagnetic field equation solver GdfidL. To explain the measured tune shifts, the impedance of each component is weighted by the local $\beta$-function. Due to the strong modulation of the optics, the $\beta$-functions play a key role in understanding the importance of the different machine elements in the impedance budget. The incoherent quadrupolar detuning is the second important effect which has to be taken into account to obtain the impedance in both planes. The resulting impedance budgets (without resistive wall part) show that in the vertical plane the RF-liners near the dipoles yield the largest contribution due to the $\beta$-value [3]. On the other hand the contribution of all tapers to the impedance is only $20 \%$ in the vertical plane, but amounts to $80 \%$ of the budget in the horizontal plane (scrapers not included). This effect could be confirmed qualitatively by the measured tune shifts and TMCI current thresholds. On the whole, values of about $5 \mathrm{M} \Omega$ vertically and $1.8 \mathrm{M} \Omega$ horizontally have been computed at zero frequency.

Standard 5 metre-long stainless steel ID vessels $(11 \mathrm{~mm}$ vertical beam stay clear) are currently being replaced by low gap ( 5 metre-long, $8 \mathrm{~mm}$ height) vacuum chambers (five NEG coated copper-coated stainless steel and one NEG coated aluminium) together with the installation of 5 in-vacuum undulators ( 2 meter long, 5.5 $\mathrm{mm}$ minimum gap). No evolution of the instability thresholds or of the quadrupolar detuning induced by the asymmetry of the chambers could be observed in multibunch mode. The reduction of the vertical aperture was compensated by the change in the resistivity of the material. This also confirms that both instability thresholds and detuning are largely governed by chambers in the achromat, due to the local high beta functions, compared to straight sections.

Vertical single bunch characteristics have not evolved, however, single bunch operation is now strongly affected in the horizontal plane. The incoherent detuning induced by the short range wake has significantly increased. The injection efficiency and saturation is directly affected by the beam blow-up occurring at high current. The horizontal transverse mode coupling instability threshold decreased from $4 \mathrm{~mA}$ in 1999 to $0.9 \mathrm{~mA}$ and the chromaticity used for operation had to be enlarged. The horizontal instability threshold now also depends on the tune and the horizontal emittance. For all these reasons, it is envisaged to reduce the maximum single current delivered to the Users to below $15 \mathrm{~mA}$.

\section{TEST OF THE SOLEIL SUPERCONDUCTING CAVITY}

A cryo-module housing two superconducting $352 \mathrm{MHz}$ strongly HOM-damped cavities has been developed within the framework of the SOLEIL project design study phase, in a collaboration agreement between CEA, CERN, ESRF and SOLEIL. In 2002 the prototype was installed on the ESRF storage ring and tested with beam at $4.5 \mathrm{~K}$, with the cavities cooled by liquid helium poured from Dewars. Four such tests were carried out at the end of scheduled shutdowns at the ESRF. In passive operation at $300 \mathrm{~K}$ or $4 \mathrm{~K}$, no instability was detected up to the maximum ESRF intensity of $200 \mathrm{~mA}$. This demonstrated the validity of the concept of an effective damping of the Higher Order Modes (HOM). The superconducting (SC) module was tested successfully in the accelerating regime with $3 \mathrm{MV}$ of accelerating voltage. A maximum power of $360 \mathrm{~kW}$ could then be transferred to $170 \mathrm{~mA}$ of beam In order to avoid disturbing the ESRF machine performance during user mode operation, the cavities were maintained detuned at room temperature in a passive regime. The heat generated by the beam was then evacuated by means of a helium gas flow, cooled by a heat exchanger. The test arrangement provoked only three beam trips over one year and was therefore almost transparent to normal ESRF operation.

With the successful prototype test, the concept of this almost HOM-free SC cavity has been validated [4]. The tests have also shown that this cavity constitutes a valuable option for a possible future upgrade at the ESRF.

\section{REFERENCES}

[1] J Chavanne, "In-vacuum undulators at the ESRF”, this conference.

[2] R Nagaoka et al., "Incoherent transverse tune shifts due to resistive wall low gap chambers", EPAC 2002.

[3] T.F.Günzel, "Evaluation of the vertical mpedance of the ESRFmachine" EPAC 2002, Paris.

[4] J Jacob et al., "Successful beam test of the SOLEIL SC HOM free cavity at the ESRF", this conference. 